(2) Open Access Full Text Article

\title{
Basal oxidative stress ratio of head and neck squamous cell carcinomas correlates with nodal metastatic spread in patients under therapy
}

This article was published in the following Dove Press journal:

OncoTargets and Therapy

9 January 2017

Number of times this article has been viewed

D Dequanter ${ }^{1,2}$

R Dok'

S Nuyts ${ }^{1,3}$

'Department of Oncology and Experimental Radiotherapy, KU Leuven - University of Leuven, Leuven, ${ }^{2}$ Department of Head and Neck Surgery, CHU Charleroi, Montigny, ${ }^{3}$ Department of Radiation Oncology, University Hospitals Leuven, Leuven, Belgium
Correspondence: S Nuyts

Department of Radiation Oncology, University Hospitals Leuven, Herestraat, 49 Bus 7003 40, 3000 Leuven, Belgium Tel +32 I6347600

Email sandra.nuyts@uzleuven.be
Background: Head and neck squamous cell carcinoma (HNSCC) is a type of cancer that is strongly associated with oxidative damage and oxidative stress. Tobacco and alcohol - sources of massive quantities of reactive oxygen species (ROS) - have been clearly identified as etiologic factors that contribute to these malignancies. Considering the role of glutathione (GSH) in ROS detoxification, we hypothesized that potential biological markers can be found in addition to the parameters of oxidative stress. In line with previous studies that emphasized the accumulation of GSH in tumor cells, in this study, we have reported a lower ratio of oxidized versus reduced GSH in head and neck tumors.

Objective: The aim of the paper was to evaluate the prognostic and clinical significance of the ratio of oxidized versus reduced GSH in patients with head and neck cancers.

Methods: Thirty-six patients with HNSCC were included in this study. The tumoral redox status was determined by measuring the ratio of oxidized/reduced GSH (GSSG/GSH) by capillary electrophoresis. Statistical analysis was performed to assess the correlation between patient, clinical factors and the redox status.

Results: The results showed a low tumoral ratio of GSSG/GSH and a better locoregional control. Moreover, a significant correlation between the tumoral redox status ratio (GSSG/GSH) and nodal stage ( $\mathrm{N} 0$ versus N1, N2 and N3) was also observed. A higher tumoral redox status ratio was found to be associated with the presence of lymph node metastasis (N1, N2 and N3).

Conclusion: A strong correlation was observed between the oxidative status and locoregional control of the tumors. Moreover, a higher basal tumoral redox status ratio was found to be correlated with the presence of lymph node metastasis.

Keywords: oxidative stress, head and neck cancer, glutathione ratio, prognostic factor, lymph node metastasis

\section{Introduction}

Recently, oxidative stress alterations have been well established as a hallmark of cancer. This factor is of particular importance in causing tumors of the head and neck, as these are strongly associated with oxidative damage and oxidative stress, with tobacco and alcohol clearly defined as major etiologic factors. Increased levels of reactive oxygen species (ROS) are commonly found in cancer cells, and cancer cells of other types also demonstrate increased sensitivity to ROS.

Cancer cells and normal cells adapt to oxidative stress by activation of multiple pathways including kinase signal transduction pathways and transcription factors, which results, among other effects, in the upregulation of genes that encode antioxidant enzymes and molecules. ${ }^{1}$ 
Among these, glutathione (GSH) is an important antioxidant because several of the antioxidant mechanisms that involve reduction reactions ultimately rely on the oxidoreduction of GSH. ${ }^{1,2}$

Low levels of intracellular GSH decrease cellular antioxidant capacity, whereas elevated levels of GSH generally increase the antioxidant capacity and resistance to oxidative stress. This phenomenon is observed in many types of cancer cells. ${ }^{2,3}$ In this study, we investigated the clinical and prognostic significance of the ratio of oxidized/reduced GSH (GSSG/GSH) in cancer tissues in a cohort of 36 head and neck cancer patients.

\section{Materials and methods}

The present study was approved by the Ethic Committee of André Vésale Hospital (OM008) under CCB number B32520107991. After obtaining approval from the committee and written informed consent of all the patients, 36 newly diagnosed head and neck squamous cell carcinoma (HNSCC) patients were prospectively included in this study. There were 6 females and 30 males and the median age was 59 years. The numbers of patients with tumor-nodemetastasis (TNM) stages I, II, III and IV were 4, 5, 3 and 24 , respectively. Patients were categorized based on tumor localization: 15 patients with tumor in larynx, 9 patients with tumor in pharynx, 8 with tumor in hypopharynx and 4 in oropharynx (Table 1). All patients were treated surgically. Radiotherapy was initiated in 10 patients $4-6$ weeks after surgery. All these patients received between 50 and 70 Gy of radiation; they were treated once a day with conventional fractionation, 5 times a week, over the course of 6 weeks. Seventeen of the 36 patients received concomitant chemoradiotherapy. Median follow-up was 63 months.

First, the surgical specimen was analyzed by an anatomopathologist to differentiate between the normal (without any presence of cancer cells) and tumor tissues. The same procedure was followed for heterogeneous tumors to exclude necrosis and to select tumoral specimen with cancerous cells by microscopy. Second, the ratio of oxidized to reduced GSH was determined using the capillary electrophoresis kit, Ceofix (Analis, Namur, Belgium), according to the manufacturer's instructions and as per the data available in the literature. ${ }^{1,2}$ Briefly, $200 \mu \mathrm{L}$ of whole blood and healthy and tumor tissues were obtained from the patient, immediately ground with $600 \mu \mathrm{L}$ of $5 \%$ metaphosphoric acid and centrifuged (within 3 hours). A total of $100 \mathrm{~mL}$ of the supernatant was mixed with $400 \mu \mathrm{L}$ of the kit diluent, containing naphthalene sulfonic acid as internal standard. The sample was analyzed using a $\mathrm{P} /$ ACE 5000 series with $37 \mathrm{~cm}$ and $75 \mu \mathrm{m}$ identified by capillary
Table I Characteristics of 36 patients

\begin{tabular}{|c|c|}
\hline Parameters & $\begin{array}{l}\text { Number of } \\
\text { patients }\end{array}$ \\
\hline \multicolumn{2}{|l|}{ Gender } \\
\hline Male & 30 \\
\hline Female & 6 \\
\hline \multicolumn{2}{|l|}{ Age, years } \\
\hline Median (range) & $59(47-8 I)$ \\
\hline \multicolumn{2}{|l|}{ Nodal stage } \\
\hline No & 20 \\
\hline $\mathrm{NI} / \mathrm{N} 2 / \mathrm{N} 3$ & 16 \\
\hline \multicolumn{2}{|l|}{ T-stage } \\
\hline $\mathrm{TI} / \mathrm{T} 2$ & 14 \\
\hline $\mathrm{T} 3 / \mathrm{T} 4$ & 22 \\
\hline \multicolumn{2}{|l|}{ Disease stage } \\
\hline I-II & 9 \\
\hline III-IV & 27 \\
\hline \multicolumn{2}{|l|}{ Tumor site } \\
\hline Hypopharynx & 8 \\
\hline Oropharynx & 4 \\
\hline Pharynx & 9 \\
\hline Larynx & 15 \\
\hline \multicolumn{2}{|l|}{ Treatment } \\
\hline RT & 10 \\
\hline $\mathrm{RT}+\mathrm{CT}$ & 17 \\
\hline No adjuvant treatment & 9 \\
\hline \multicolumn{2}{|l|}{ Redox potential } \\
\hline Mean GSSG/GSH tumor (range) & $6.69(0.88-46.6)$ \\
\hline
\end{tabular}

Abbreviations: RT, radiotherapy; RT + CT, concomitant chemoradiotherapy; GSSG/GSH, oxidized/reduced glutathione.

maintained at $25^{\circ} \mathrm{C}$. Separation of the contents was performed at $8 \mathrm{kV}$ with borate buffer at $\mathrm{pH} 8.2$ containing SDS. The peaks of oxidized and reduced GSH were detected at $200 \mathrm{~nm}$ and integrated. The total integrated area of the oxidized GSH peak was then divided by that of the reduced one.

Cox proportional hazard analysis was performed to assess the relation between redox status (oxidized/reduced glutathione $[\mathrm{GSSG} / \mathrm{GSH}]$ ) of the untreated tumor biopsies and clinical end points such as locoregional control (LRC), overall survival (OS) and disease-specific survival (DSS). OS was defined as the time between the start of treatment and death; DSS as the time between the start of treatment and disease-specific recurrence or death; and LRC as the time between the start of treatment and date of locoregional recurrence. Correlation between clinical/patient characteristics and redox status was assessed using ANOVA. The redox status was defined as the ratio of GSSG and GSH and was assessed as a continuous variable. The following conditions were assessed as a binary variable: nodal stage - absence of nodal involvement (N0) and presence of nodal involvement (N1, N2 and N3); T-stage - lower T-stages (T1 and T2) and higher T-stages (T3 and T4); and disease stage - lower disease stages (I and II) and higher disease stages (III and IV) 
(Table S1). All analyses were 2-sided and performed using Statistica software (version 12 ). $P$-values $\leq 5 \%$ were considered significant for all tests.

\section{Results}

Histological examination of the surgical specimens showed the presence of squamous cell carcinomas in all cases: poorly differentiated in 4 cases $(11 \%)$, moderately differentiated in 14 cases (39\%) and well differentiated in 18 cases $(50 \%)$.

The results were expressed as delta values, that is, the difference between the normal mucosa and tumor tissues which highlights the variability among the samples. The results obtained showed that 21 patients had a lower tumoral ratio of GSSG/GSH compared with normal tissues $(58.3 \%)$, whereas 15 patients $(41.7 \%)$ had a higher tumoral GSSG/GSH ratio. ${ }^{3}$

Oxidative stress ratio obtained by Cox regression analysis showed no significant difference in the OS. A strong correlation was observed between the LRC rates of the tumors and their oxidative status (hazard ratio $=0.69$ ) (confidence interval $[\mathrm{CI}]$ : $0.44-1.08 ; P=0.1)$. Moreover, univariate analyses showed a significant association between the tumoral redox status ratio (GSSG/GSH) and nodal stage (N0 versus N1, $\mathrm{N} 2$ and N3), with a higher tumoral redox status ratio associated with the presence of lymph node metastasis (N1, N2 and N3) (Figure 1). No significant correlation was observed between tumoral GSSG/GSH and disease stage and T-stage.

\section{Discussion}

Head and neck cancer cells adapt to oxidative stress by activation of multiple pathways. Among the different salvage

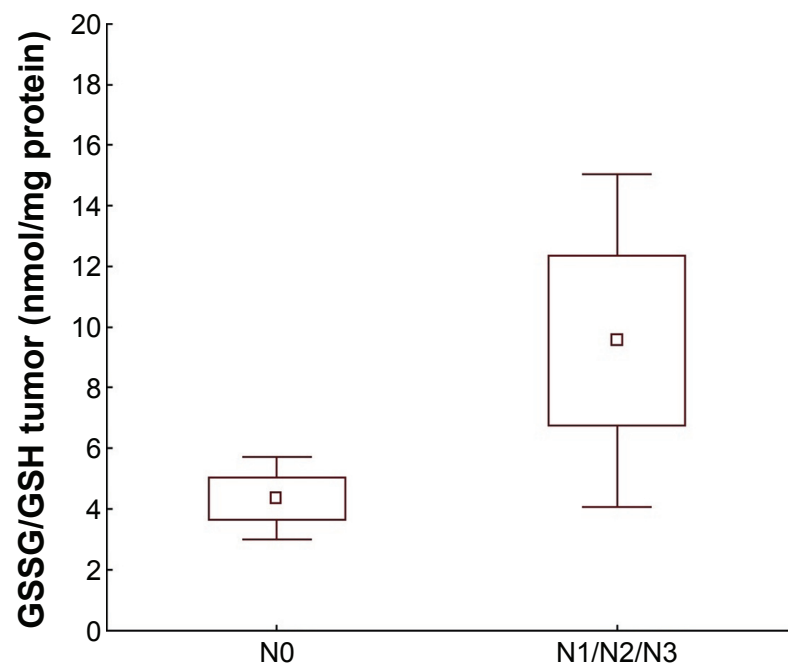

Figure I Box plot showing the mean values of the ratio of oxidized and reduced glutathione (GSSG/GSH) (with 95\% confidence interval) for nodal stages indicated (N0 versus $\mathrm{NI} / \mathrm{N} 2 / \mathrm{N} 3$ ), $P=0.05$. pathways, GSH plays a key role in ROS detoxification. GSH is found in two forms: free or bound to proteins. The free form is present mainly in its reduced form (GSH), which is converted to its oxidized form (GSSG) during oxidative stress. $^{4}$

Oxidative damage is prevented by the reduction of GSSG to GSH in the presence of GSSG reductase at the expense of NADPH, which results in a redox cycle. Organic peroxides can be reduced both by glutathione peroxidase $\mathrm{X}$ and GSHtransferase (GST). In extreme oxidative stress conditions, the ability of the cell to reduce GSSG to GSH is decreased, thereby resulting in the accumulation of GSSG within the cytosol. To avoid a shift in the redox equilibrium, the GSSG is actively transported out of the cell or it is reacted with protein sulfhydryl groups.

Therefore, in this study, we focused on the ratio of GSSG/ GSH because the antioxidant cellular armada involves cascades of oxidoreduction, which ultimately result in oxidized/ reduced GSH. Furthermore, the equilibrium of this reaction depends on the concentrations of GSH and GSSG, and their molar ratios are indicators of cell functionality and oxidative stress. $^{4}$

However, we have previously demonstrated a large variability in the tumoral concentrations of GSH and GSSG which is in agreement with the results reported in the literature (from $0.69 \pm 0.73 \mu \mathrm{mol} / \mathrm{g}$ tissue to $30.14 \pm 6.37 \mathrm{mg} / \mathrm{mg}$ protein). ${ }^{3}$ Moreover, we observed that a considerable proportion $(58.3 \%)$ of tumor patients exhibited a lower ratio of GSSG/GSH compared to healthy individuals. These results, also observed for tumors of other types, are consistent with those found in the literature. ${ }^{4,5}$

It is also well known that any change in the intracellular GSSG/GSH ratio results in the activation of cell proliferation and cell death. Little is known, however, about the clinical and the prognostic significance of the ratio GSSG/GSH in tumor tissues.

In this study, we have investigated the prognostic significance of the tumoral ratio of GSSG/GSH by assessing the correlation between oxidized/reduced GSH in tumoral tissue and clinical parameters of head and neck cancer patients.

Few studies have evaluated the correlation between clinicopathological parameters and GSH. Two studies found no correlation between GSH levels and the degree of differentiation. ${ }^{6,7}$ However, Wong et al found higher levels of GSH in well differentiated oral carcinoma compared with moderately differentiated tumors. ${ }^{8}$ Kolanjiappan et al and Parise et al reported increasing GSH levels with tumor stage. ${ }^{9,10}$ This correlation was not found by Guichard et $\mathrm{al}^{6}$ 
but they limited their study to primarily T2 and T3 patients. ${ }^{11}$ Similarly, Inci et al found no difference in GSH levels when comparing patients grouped as either stage I/II or stage III. ${ }^{12}$ Likewise, Wong et al found no correlation between GSH levels and the tumor size in oral carcinoma and Lafuente et al found no correlation in laryngeal tumors. ${ }^{8,13}$ In addition, some authors reported that GSH level increased with tumor size, mainly with the nodal status. ${ }^{14}$

In this study, we observed a strong correlation between the levels of oxidized and reduced GSH in tumor tissues and the nodal status of the head and neck cancer patients. A lower GSSG/GSH ratio was observed in tumor tissues of N0 patients, while a higher GSSG/GSH ratio was observed in positive node patients. This suggests that tumors with a higher GSSG/GSH ratio might have a tendency to spread more locally, due to their more aggressive phenotype caused by oxidative stress. However, the exact role of GSH in the prognosis of HNSCC is not fully understood. Previous studies have indicated that HNSCC patients with a lower GSH level may be highly sensitive to antitumor treatments, which may result in a better outcome, whereas increased GSH levels are associated with drug resistance and poor prognosis. ${ }^{1,15}$

To date, no studies have evaluated the relationship between patient outcome and GSH measurements. ${ }^{8}$ The most promising result of our study is that patients with a lower GSSG/GSH ratio compared to those with higher ratio had a lower risk of local and regional recurrence of tumor after treatment. This suggests a more aggressive behavior of the tumors with high oxidative stress.

\section{Limitations}

Our study has few limitations. The sample size included in our study is small, which is a common problem to many studies related to head and neck tumors, and is due to their heterogeneity. Another limitation is the lack of multivariate analysis, due to the relatively small number of patients included.

\section{Conclusion}

Despite these limitations, we confirmed a correlation between higher tumoral ratio of GSSG/GSH and the initial spread of nodal metastasis. This result shows that a lower tumoral GSSG/GSH ratio at the time of the initial treatment has a higher tendency for LRC. This observation suggests that pretherapeutic tumoral ratio of GSSG/GSH is of prognostic value. However, a prospective study with large patient population should be carried out to further validate these findings.

\section{Disclosure}

The authors report no conflicts of interest in this work.

\section{References}

1. Abdalla MY. Glutathione as potential target for cancer; more or less is good? JJBS. 2011;4(3):119-124.

2. Ames BN. Endogenous oxidative DNA damage, aging, and cancer. Free Radic Res Commun. 1989;7(3-6):121-128.

3. Dequanter D, Van de Velde M, Nuyens V, et al. Assessment of oxidative stress in tumors and histologically normal mucosa from patients with head and neck squamous cell carcinoma: a preliminary study. Eur $J$ Cancer Prev. 2013;22(6):558-560.

4. Fiaschi AI, Cozzolino A, Ruggiero G, Giorgio G. Glutathione, ascorbic acid and antioxidant enzymes in the tumor tissue and blood patients with oral squamous cell carcinoma. Eur Rev Med Pharmacol Sci. 2005; 9(6):361-367.

5. Gamcsik MP, Kasibhatla MS, Teeter SD, Colvin OM. Glutathione levels in human tumors. Biomarkers. 2012;17(8):671-691.

6. Guichard M, Lespinasse F, Estelin R, et al. Glutathione and cysteine levels in human tumor biopsies Br J Radiol. 1990;63(751):557-561.

7. Havel KL, Pritts K, Wielgros T. Quantification of oxidized and reduced glutathione in plasma by micellar electrokinetic capillary electrophoresis. J Chromatogr A. 1999;853(1-2):215-223.

8. Wong DY, Hsiao YL, Poon CK, et al. Glutathione concentration in oral cancer tissues. Cancer Lett. 1994;81(2):111-116.

9. Kolanjiappan K, Ramachandran CR, Manoharan S. Biochemical changes in tumor tissues of oral cancer patients. Clin Biochem. 2003;36: 61-65.

10. Parise O Jr, Janot F, Luboinski B, et al. Thymidylate synthase activity, folates, and glutathione system in head and neck carcinoma and adjacent tissues. Head Neck. 1994;16(2):158-164.

11. Monostori P, Wittman G, Karg E, Turi S. Determination of glutathione and glutathione disulfide in biological samples; an in-depth review. J Chromatogr B Analyt Technol Biomed Life Sci. 2009;877: 3331-3346.

12. Inci E, Civelek S, Seven A, Inci F, Korkut N, Burcak G. Laryngeal cancer: in relation to oxidative stress. Tohoku J Exp Med. 2003;200:17-23.

13. Lafuente A, Maristany M, Arias C, et al. Glutathione and glutathione S-transferases in human squamous cell carcinomas of the larynx and GSTM1 dependent risk. Anticancer Res. 1998;18(1A):107-111.

14. Patel BP, Rawal UM, Dave TK, et al. Lipid peroxidation, total antioxidant status and total thiol levels predict overall survival in patients with oral squamous cell carcinoma. Integr Cancer Ther. 2007;6(4):365-372.

15. Mulcaly RT, Untawole S, Gipp JJ. Transcriptional upregulation of gamma-glutamyl cysteine synthetase gene expression in melphalanresistant human prostate carcinoma cells. Mol Pharmacol. 1994;46(5): 909-914. 


\section{Supplementary material}

Table SI Distribution of patients according to nodal stage

\begin{tabular}{|c|c|c|c|}
\hline $\mathrm{N}$-stage & No & $\mathrm{N} 1 / \mathrm{N} 2 / \mathrm{N} 3$ & Total \\
\hline Number of patients ( $n$ ) & 20 & 16 & 36 \\
\hline \multicolumn{4}{|l|}{ Age, years } \\
\hline Mean & 61.8 & 57.8 & \\
\hline \multicolumn{4}{|l|}{ Gender } \\
\hline Male & 18 & 12 & 30 \\
\hline Female & 2 & 4 & 6 \\
\hline \multicolumn{4}{|l|}{ Localization } \\
\hline Larynx & 10 & 5 & 15 \\
\hline Hypopharynx & 2 & 6 & 8 \\
\hline Pharynx & 4 & 5 & 9 \\
\hline Oropharynx & 4 & 0 & 4 \\
\hline \multicolumn{4}{|l|}{ T-stage } \\
\hline $\mathrm{TI} / \mathrm{T} 2$ & 10 & 4 & 14 \\
\hline $\mathrm{T} 3 / \mathrm{T} 4$ & 10 & 12 & 22 \\
\hline \multicolumn{4}{|l|}{ Disease stage } \\
\hline I-II & 10 & 4 & 14 \\
\hline III-IV & 10 & 12 & 22 \\
\hline \multicolumn{4}{|l|}{ Tumor site } \\
\hline Larynx & 10 & 5 & 15 \\
\hline Hypopharynx & 2 & 6 & 8 \\
\hline Pharynx & 4 & 5 & 9 \\
\hline Oropharynx & 4 & 0 & 4 \\
\hline \multicolumn{4}{|l|}{ Redox potential } \\
\hline Mean GSSG/GSH tumor & 4.3 & 9.5 & 6.6 \\
\hline
\end{tabular}

Abbreviation: GSSG/GSH, oxidized and reduced glutathione.

\section{Publish your work in this journal}

OncoTargets and Therapy is an international, peer-reviewed, open access journal focusing on the pathological basis of all cancers, potential targets for therapy and treatment protocols employed to improve the management of cancer patients. The journal also focuses on the impact of management programs and new therapeutic agents and protocols on

\section{Dovepress}

patient perspectives such as quality of life, adherence and satisfaction. The manuscript management system is completely online and includes a very quick and fair peer-review system, which is all easy to use. Visit http://www.dovepress.com/testimonials.php to read real quotes from published authors. 